\title{
MODELLING AND OPTIMISATION OF RAILWAY CREW SCHEDULING
}

\author{
ROSMALINA HANAFI
}

(Received 24 April 2015; first published online 8 July 2015)

2010 Mathematics subject classification: primary 90B35; secondary 90C11, 90C59.

Keywords and phrases: crew scheduling, railways, mixed integer programming, constraint programming, heuristic methods.

Crew scheduling is one stage of operational planning in transportation systems to which mathematical and algorithmic optimisation techniques can be applied. The crew scheduling problem (CSP) in the transportation industry represents a computationally difficult combinatorial optimisation problem. The large number of tasks (trips) to include and the complicated operational and contractual requirements are the main reasons for the complexity of the problem. Nevertheless, solving CSP has been one of the most important focuses of the transportation industry because it affects the company's profitability and its service quality. An optimal crew schedule is essential to ensure efficient and reliable operations of transportation services. Furthermore, the cyclic nature of the crew scheduling application makes CSP a good candidate for optimisation. A small improvement to the crew schedules can lead to accumulated savings that produce large annual cost savings. The difficulty of solving CSP and its enormous practical significance have led to a large number of proposed solution techniques. However, unlike CSP in other modes of transportation, such as airlines and buses which have been intensively studied, railway CSP is less cited in the literature. Railway crew scheduling is domain specific and no solving method has been developed that has been applied universally. Models and algorithms are designed mainly for a specific case and may not readily be applied in different applications.

Railway CSP is the process of allocating train services to the crew duties based on the published train timetable while satisfying operational and contractual requirements. The problem is restricted by many constraints and it belongs to the class of NP-hard (nondeterministic polynomial-time hard). CSP is most frequently formulated mathematically, as either a set-covering problem or a set-partitioning

Thesis submitted to Queensland University of Technology in October 2014; degree approved on 11 November 2014; supervisor Erhan Kozan.

(c) 2015 Australian Mathematical Publishing Association Inc. 0004-9727/2015 \$16.00 
problem, and then solved analytically or approximately. Even though some studies have been done on CSP using a wide variety of solution techniques, the problem is still difficult to solve (see, for example, [1, 2]). CSP involves real-life constraints which are difficult to handle, such as crew breaks, elapsed time and the requirement to return the crews to their home depots at the end of their duty. Furthermore, an optimisation model should be well designed so that all the relevant parameters of the problem can be incorporated. One way of dealing with the problem is to develop a specific model that is capable of incorporating important features of the problem and that can be solved using a wide range of methods.

This research has developed and analysed two railway crew scheduling models. The first is a mathematical programming (MP)-based model which is formulated as a mixed integer programming (MIP) problem, while the second is a constraint programming (CP)-based model. The objective of the optimisation models is to minimise the number of crew duties by reducing idle transition times. Duties are generated by arranging scheduled trips over a set of duties and sequentially ordering the set of trips within each set of duties. The integration of a relief opportunities period (ROP) into the models enables the train crew to be relieved at any relief point (RP) within the interval of ROP. Existing models and algorithms usually consider relieving a crew only at the beginning of the interval of relief opportunities, which may be impractical. The inclusion of the ROP into models has not been studied in depth. Allowing the train crew to be relieved at any RP during the ROP will provide better representation of real-world conditions and improve the robustness of the schedule.

Due to the combinatorial nature of the CSP, heuristic methods are the most promising approach for solving the problem. The main limitation with many of the conventional heuristic algorithms is their difficulty to escape from locally optimal solutions. The search is usually conducted from a single point in the solution space and then continues until there is no possible improvement. This type of local search method can easily get trapped in local optima. In an attempt to address this problem, several metaheuristics approaches have emerged for solutions to combinatorial complex problems such as simulated annealing (SA) and tabu search (TS). SA and TS have been applied successfully to solve many combinatorial optimisation problems in various practical settings. Despite the potential application of metaheuristics to solve combinatorial optimisation problems, very few attempts have been made to tackle crew scheduling related problems in the literature applying metaheuristics.

SA and TS algorithms have been utilised in this study to improve solutions and to derive near-optimal solutions. An initial solution for railway CSP is generated by a constructive heuristic $(\mathrm{CH})$ and then it is improved by a hybrid constructive heuristic SA (HCHSA) algorithm. Both the $\mathrm{CH}$ and the HCHSA algorithms produced acceptable solutions, although the solutions produced are not guaranteed to be optimal. The HCHSA algorithm significantly improves the solution produced by the $\mathrm{CH}$. The HCHSA algorithm increases the average driving time by $3.06 \%$ and decreases the average excess cost by $3.35 \%$. Overall, the HCHSA algorithm increases the total crew working time and reduces the number of crew duties for all datasets. As the 
number of crew duties corresponds to the number of crew needed, significant savings can be gained on the annual cost of crew related expenses. The solutions produced by the hybrid constructive heuristic TS algorithm, which was composed of a three-phase heuristic, indicate that the proposed algorithm is able to generate near-optimal feasible solutions within an acceptable computational time. This is indicated by the average $Q$ value, which is fairly close to zero. An aggregation procedure has a significant effect in reducing the problem size such that the proposed TS-based algorithms are able to handle large-sized railway CSP and solve it within an acceptable computational time. The neighbourhood structure also contributes to the effectiveness of the search process. The solutions obtained by the hybrid CP and SA algorithm and the hybrid TS and SA algorithm also give an indication of the effectiveness of hybridisation of an exact method and metaheuristics as well as hybridisation of metaheuristics (TS and SA) to produce good acceptable solutions in a reasonable computational time for large instances (see [3]).

\section{References}

[1] R. L. Burdett and E. Kozan, 'A disjunctive graph model and framework for constructing new train schedules', Eur. J. Oper. Res. 200(1) (2010), 85-98.

[2] C. Goumopoulos and E. Housos, 'Efficient trip generation with a rule modeling system for crew scheduling problems', J. Syst. Softw. 69(1-2) (2004), 43-56.

[3] R. Hanafi and E. Kozan, 'A hybrid constructive heuristic and simulated annealing for railway crew scheduling', Comput. Ind. Eng. 70 (2014), 11-19.

ROSMALINA HANAFI, Science and Engineering Faculty,

Queensland University of Technology, Brisbane,

Queensland 4001, Australia

e-mail: rosmalina.hanafi@hdr.qut.edu.au 